\title{
Logistics Reduction and Repurposing Beyond Low Earth Orbit
}

\author{
James Lee Broyan, $\mathrm{Jr}^{1}$ and Michael K. Ewert. ${ }^{2}$ \\ NASA Lyndon B. Johnson Space Center, Houston, Texas, 77058
}

\begin{abstract}
All human space missions, regardless of destination, require significant logistical mass and volume that is strongly proportional to mission duration. Anything that can be done to reduce initial mass and volume of supplies or reuse items that have been launched will be very valuable. Often, the logistical items require disposal and represent a trash burden. Utilizing systems engineering to analyze logistics from cradle-to-grave and then to potential reuse, can minimize logistics contributions to total mission architecture mass. In NASA's Advanced Exploration Systems "Logistics Reduction and Repurposing Project”, various tasks will reduce the intrinsic mass of logistical packaging, enable reuse and repurposing of logistical packaging and carriers for other habitation, life support, crew health, and propulsion functions, and reduce or eliminate the nuisances aspects of trash at the same time. Repurposing reduces the trash burden and eliminates the need for hardware whose function can be provided by use of spent logistic items. However, these reuse functions need to be identified and built into future logical systems to enable them to effectively have a secondary function. These technologies and innovations will help future logistic systems to support multiple exploration missions much more efficiently.
\end{abstract}

${ }^{1}$ AES Logistics Reduction Project Manager, Crew \& Thermal Systems Division, M/S: EC3, not AIAA affiliated.

${ }^{2}$ AES Logistics Reduction SE\&I Lead, Crew \& Thermal Systems Division, M/S: EC2, not AIAA affiliated. 